\title{
A geometrical model to predict the wear evolution of coated surfaces
}

\author{
A. Ramalho* \\ ICEMS, Department of Mechanical Engineering, University of Coimbra, \\ Pinhal de Marrocos, P-3030 Coimbra, Portugal \\ Accepted 21 December 2006 \\ Available online 14 June 2007
}

\begin{abstract}
The improvement of the thin films production techniques leads to the availability of a wide range of coatings with high mechanical properties difficult to be reached with monolithic materials. Thin coated surfaces reveal promising results in several applications, especially where high wear resistance is required. However, selecting the best solution for an envisaged application is a difficult task because the tribological performance depends on both coating and substrate properties and also on adhesion between the coating and the substrate. Therefore, among the main challenges of the surface engineering are included suitable procedures to assure enough adhesion between the coating and the substrate and calculation methods to predict the wear and the mechanical behaviour of each coating + substrate arrangement. Ball-cratering micro-scale abrasion technique solve partially this problem once it allows to determine the specific wear rates of coating and substrate by only one set of tests done with the coated surfaces. In this paper, prediction models based on the micro-scale abrasion tests, will be presented and discussed. The derived geometrical models allow the study of ball-on-plane contact and also of crossed-cylinders contact. The ability of these models to be applied on coating development was demonstrated applying the prediction criteria to study the effect of coating thickness and the coating intrinsic specific wear rate. The wear evolution forecasted by the model was also compared to experimental results of hard chromium plated steel and very good correlations were reached.
\end{abstract}

(C) 2007 Elsevier B.V. All rights reserved.

Keywords: Coatings; Wear; Abrasion; Prediction models

\section{Introduction}

Nowadays, thin coatings are widely used to improve the mechanical properties, particularly wear resistance, of the effective surface of engineering parts. Ball-cratering is now a well-established technique for characterizing thin coated materials. As curved shapes, particularly cylindrical ones, are widely used in mechanical applications, the characterization of coated cylindrical shapes by micro-abrasion could enlarge the application of the technique. A recent paper demonstrates that crossed-cylinder geometry can be used to micro-abrasion testing of cylindrical shapes [1].

The development of thin hard coatings for tribological applications usually requires a long experimental study to optimize the substrate/film response for the envisaged application. How-

\footnotetext{
* Tel.: +351 239 790756; fax: +351239790701.

E-mail address: amilcar.ramalho@dem.uc.pt.
}

ever, each time more knowledge is available to aid the coating selection [2-4].

This paper focuses the development of a complementary model to predict the behaviour of cylindrical shaped surfaces. The ability of these models to be applied on coating development will be demonstrated applying the prediction criteria to study the effect of coating thickness and the coating intrinsic specific wear rate.

\section{Theoretical concepts}

In the crossed cylinder micro-abrasion arrangement a cylindrical coated specimen is tested against a rotating shaft. The crossed cylinder micro-abrasion test is an imposed shape wear test where the wear scar is very small and the contact is fixed in the specimen and is circumferential in the moving shaft. Kassman et al. [5] and Rutherford and Hutchings [6] have derived the main theoretical concepts of ball-cratering as a 
micro-scale abrasive test for coated materials. Ramalho [1] extended and updated the principle to be applied to cross cylinder geometry.

Archard's wear model present the wear volume $(V)$ proportional to the normal load $(N)$, the sliding distance $(S)$ and the inverse of the hardness $(H)$. The wear coefficient $K$ establishes the proportionality and can be used as a measure of material wear behaviour. Both $K$ and $H$ include the material response and can thus, as proposed by Czichos [7], be grouped in a parameter, usually called the specific wear rate, $k$, that can be used instead the wear coefficient $K$, giving the following equation:

$V=k S N$

For bulk materials the specific wear rate can be easily calculated because the normal applied load is transferred totally by the wear scar. However, the study of coated materials raises some specific problems, mainly with respect to thin coatings. The testing of thin coatings usually leads to wear scars through the coating, with a bull-eye type pattern, and a mixed contact, coating + substrate, is involved. In general, it is expected that the coating and the substrate exhibit different $k$ values, therefore the wear behaviour depends on the response of both the coating and the substrate.

As introduced by Kassman [5], the Archard wear model, can be applied to the wear of coatings with composite contacts, i.e. wear scars that include areas of both substrate and coating materials. In general, it is expected that the coating and the substrate exhibit different $k$ values; so, the wear behaviour depends on the response of both the coating and the substrate. It is assumed that micro-scale abrasion occurs by equal wear principle. Therefore, the wear growth by imposed shape, keeping constant the geometry of the scar which counterform the rotating cylinder (or sphere in the case of ball-cratering). For coating materials, the load is partially supported by the coating and the substrate. $N_{\mathrm{c}}$ and $N_{\mathrm{S}}$ are the amount of load supported, respectively by the coating and the substrate which verify Eq. (2). Eq. (1) can be applied separately to the substrate and the coating parts of the wear scar. Finally taking into account Eq. (2) and as the sliding distance $S$ is the same for both the coating and the substrate, Eq. (3) can be derived:

$N=N_{\mathrm{c}}+N_{\mathrm{s}}$

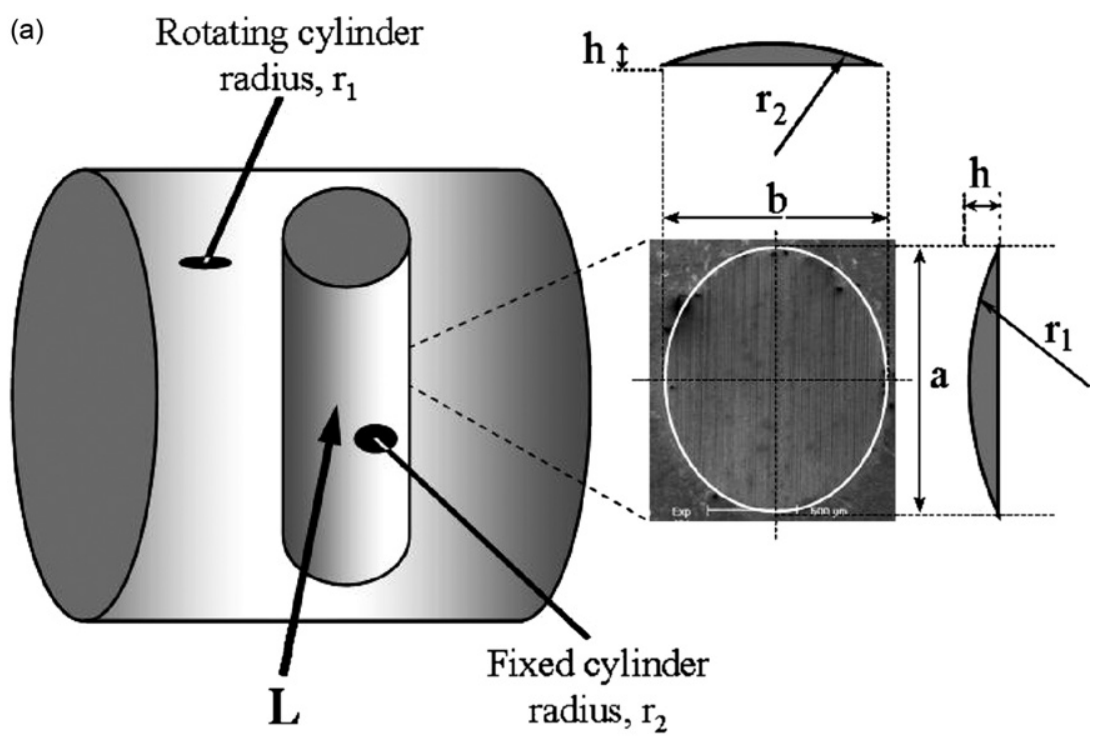

(b)

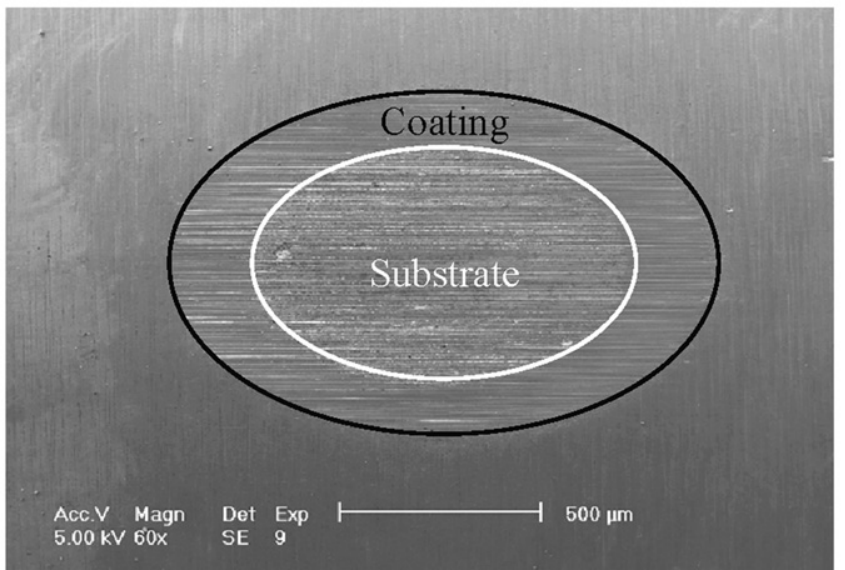

Fig. 1. (a) Contact geometry and wear scar on cross-cylinder micro-scale abrasion. (b) Typical scar obtained by testing coated specimens. 
$S N=\frac{V_{\mathrm{s}}}{k_{\mathrm{s}}}+\frac{V_{\mathrm{c}}}{k_{\mathrm{c}}}$

In Eq. (3) $k_{\mathrm{s}}, V_{\mathrm{s}}$ and $k_{\mathrm{c}}, V_{\mathrm{c}}$ are respectively the specific wear rates and wear volumes of the substrate and of the coating. The main differences between the several methods of results analysis usually used to find the values of $k_{\mathrm{s}}$ and $k_{\mathrm{c}}$ are concerned with how Eq. (3) is arranged to explain the specific wear rates as a function of the wear volumes [8].

The wear scar resulting from a cylindrical coated surface is shown in Fig. 1(a). For coated specimens, the central part of the area shows the exposed substrate, while the surrounding elliptical annulus corresponds to the wear of the film (Fig. 1(b)). It is thus possible, after each test, to measure both the scar corresponding to the surface of the coating, which includes all the wearing material (film + substrate), and therefore will be called the composite scar, and the scar corresponding to the film/substrate interface, which characterizes the substrate wear. Each scar is measured by taking the dimensions of the larger, $a$, and the smaller, $b$, axis of the elliptical wear surface. Considering $r_{1}$ and $r_{2}$ to be respectively the radii of the larger and the smaller contact cylinders, the value of scar depth, $h$, can be calculated by the following equation:

$h=r_{1}-\sqrt{r_{1}^{2}-\left(\frac{a}{2}\right)^{2}}=r_{2}-\sqrt{r_{2}^{2}-\left(\frac{b}{2}\right)^{2}}$

The wear volume can be calculated afterwards using the following approximate equation, which was derived by Ramalho [1]:

$V=\pi h^{2} \sqrt{r_{1} r_{2}}$

Eqs. (4) and (5) are applied either to the composite scar and the substrate scar, allowing to calculate, respectively the total wear volume $\left(V_{\mathrm{t}}\right)$ and the substrate wear volume $\left(V_{\mathrm{s}}\right)$.

The aim of this paper concerns the establishment of a prediction criterion that allows forecasts evolution of wear volumes, and wear depths, for any coating/substrate arrangement under crossed cylinder contact. The prediction criterion is based on the general formulation previously defined assuming that even for perforating tests the development of the scar is always by imposed shape wear and is similar to the procedure previously developed for ball-cratering geometry [2].

To apply the prediction model, the thickness of the coating and the specific wear rates $k_{\mathrm{s}}$ and $k_{\mathrm{c}}$ must be known. The prediction model is summarized on flow-chart displayed in Fig. 2, and could be applied step-by-step according to the following procedure:

(1) Select the maximum depth, $h_{\max }$, of the wear scar and the step $\Delta h$ to be used on the analysis.

(2) The prediction criteria starts with a first total depth of the scar, $\Delta h$, which is smaller than the coating thickness, $t$.

(3) Assuming that the axis of the elliptical scar are equal to the radius of the contacting cylinders, the values of the scar volume can be calculated (Eq. (5)). The wear volume of the substrate remains zero while $h<t$.

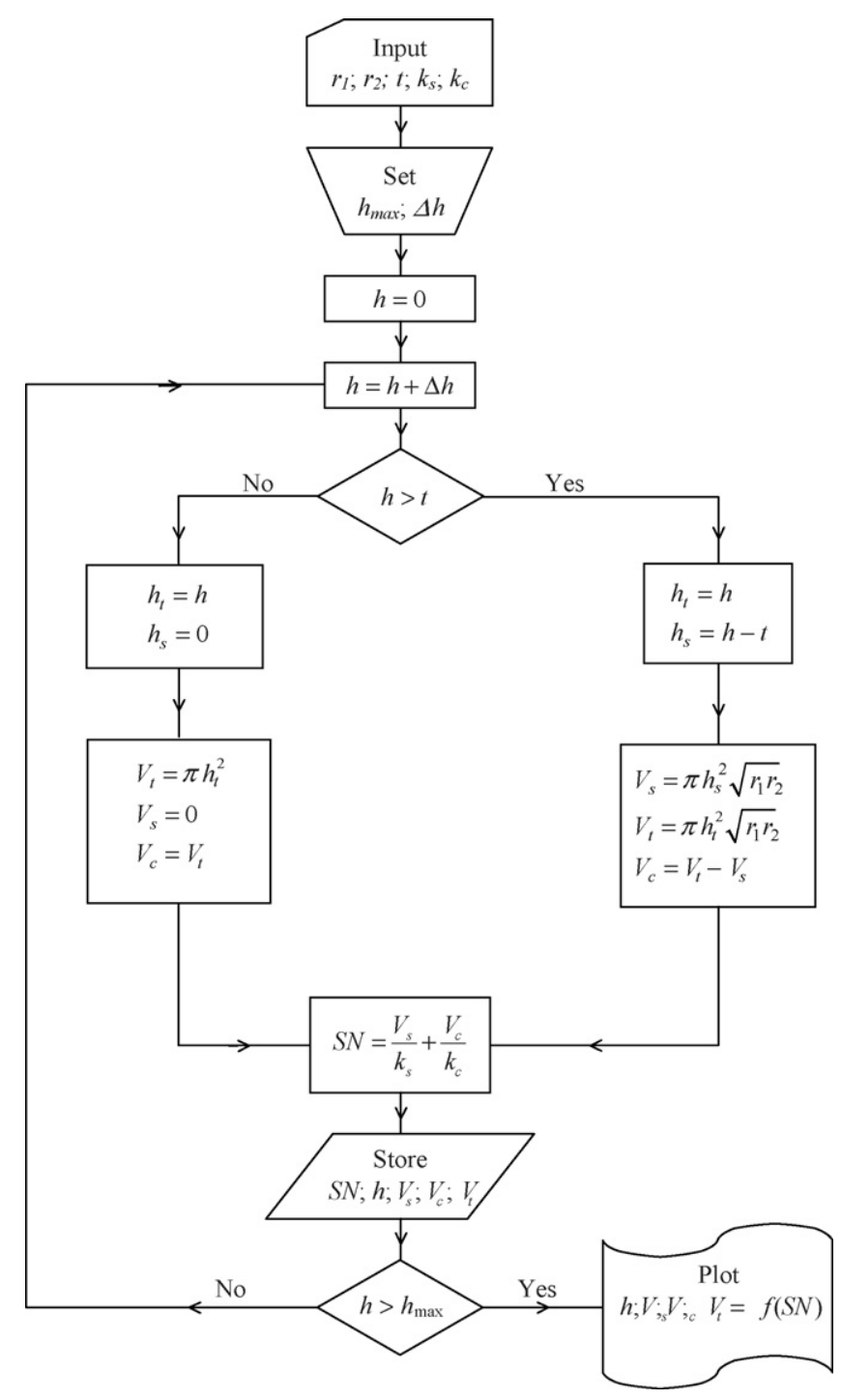

Fig. 2. Flowchart of the cross-cylinder micro-scale abrasion prediction method.

(4) Applying Eq. (5), the total wear volume can be achieved. As $h<t$ then $V_{\mathrm{c}}=V_{\mathrm{t}}$ and $V_{\mathrm{s}}=0$.

(5) As the coating specific wear rate has already been found, Eq. (2) allows to calculate the value of $S N$.

(6) Increasing progressively the scar depth, $h$, with the step $\Delta h$, for all practical values of interest, generates new values for $V_{\mathrm{c}}$ and $V_{\mathrm{t}}$.

(7) When $h>t$, the depth $h_{\mathrm{s}}$ of the scar on the substrate can be calculated as $(h-t)$.

(8) Applying Eq. (5) to the substrate and to the total wear depth, the substrate and the total wear volumes, $V_{\mathrm{s}}, V_{\mathrm{t}}$ can be found.

(9) The coating wear volume can be achieved subtracting $V_{\mathrm{S}}$ to $V_{\mathrm{t}}$.

(10) $V_{\mathrm{s}}$ and $V_{\mathrm{c}}$, can be substituted in Eq. (2) allowing to calculate $S N$.

As explained above the model allows the calculation of the relationships between the $S N$ values and the wear volumes or 
between $S N$ and the total wear depth. Calculating those values, the graphs with the predictions can be drawn.

\section{Applying the prediction model}

To verify the ability of the model to forecast the wear in specific applications, Fig. 3 shows the results obtained by applying the model to study the effect of film thickness. Fig. 3(a) shows the results obtained applying the model developed in the present paper, whereas Fig. 3(b) displays the results considering an analogous model for ball-on-plane contact [2]. The case modelled corresponds to specific wear rates of $2.5 \times 10^{-4}$ and $5 \times 10^{-3} \mathrm{~mm}^{3} / \mathrm{Nm}$, respectively, for the coating and for the substrate; the coating thickness was ranged from 1 to $6 \mu \mathrm{m}$. The results show that in spite of the continuous decreasing of wear depth verified when the thickness is increased, the efficiency of the coating is much more evident for values of thickness up to $2.5 \mu \mathrm{m}$.

Another case study is showed in Fig. 4 concerning the effect of the specific wear rate of the coating material. The substrate considered has a specific wear rate, $k_{\mathrm{s}}$, of $5.0 \times 10^{-3} \mathrm{~mm}^{3} / \mathrm{Nm}$, and all the films have a thickness of $4 \mu \mathrm{m}$. In order to evaluate the effect of the coating specific wear rate, six different values of the relationship $k_{\mathrm{c}} / k_{\mathrm{S}}$ were studied, namely $2,1,0.5,0.2,0.1$ and 0.05 . For both ball-on-plane and cross-cylinder contacts the
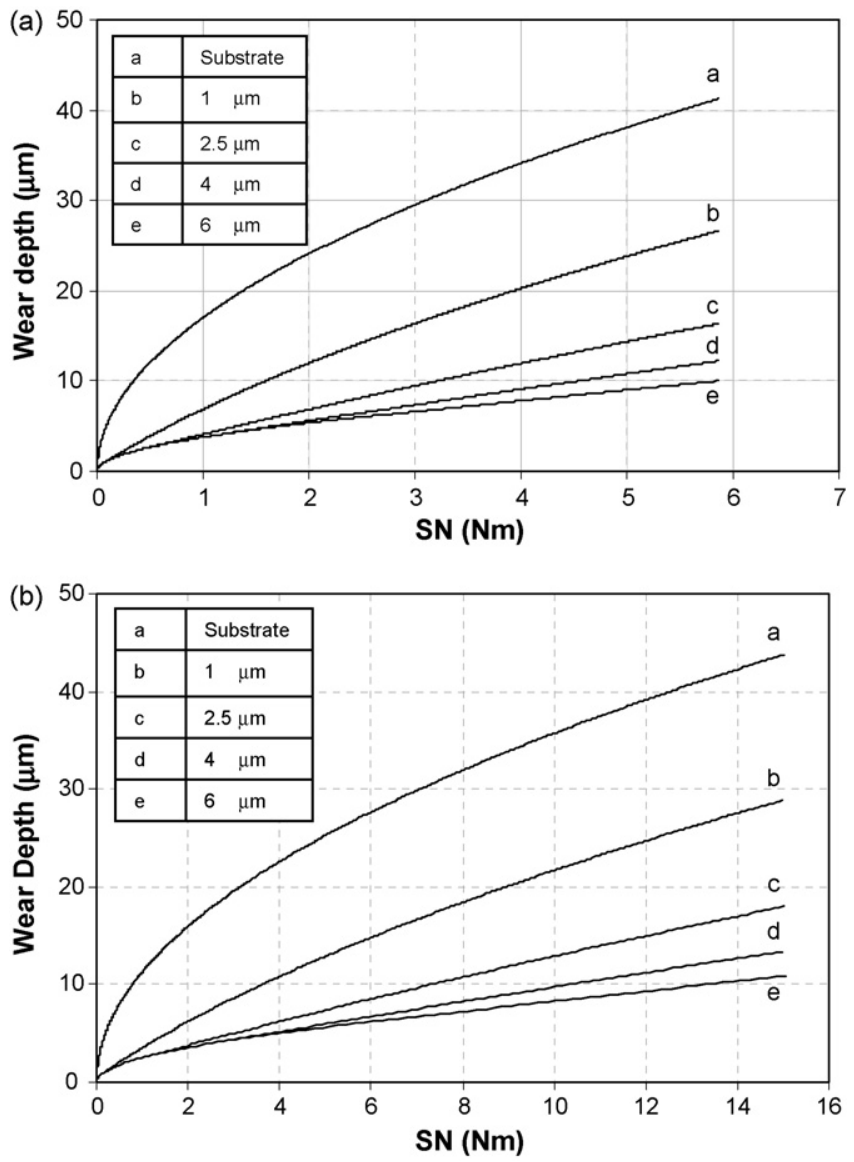

Fig. 3. Predicted results for the effect of the coating thickness. (a) Crossedcylinders contact and (b) ball-on-plane contact.
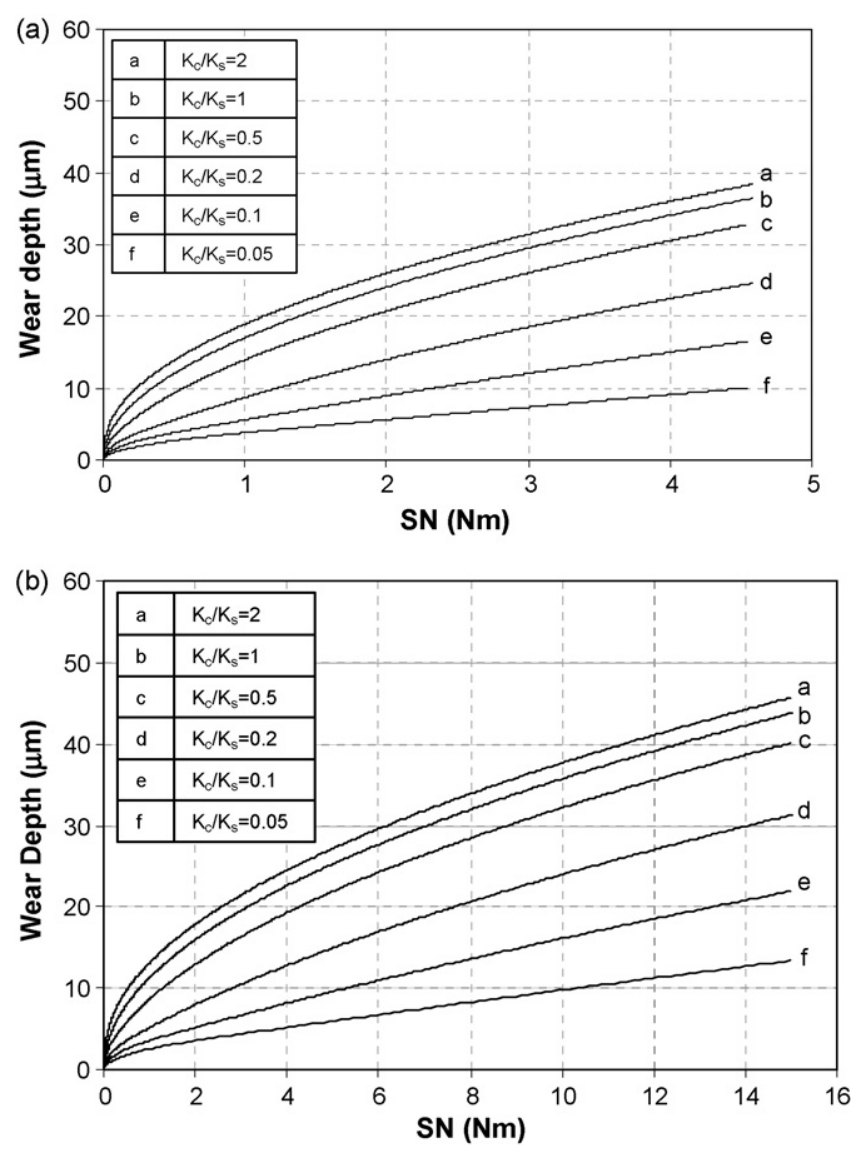

Fig. 4. Predicted results for the effect of the coating specific wear rate. (a) Cross-cylinders contact and (b) ball-on-plane contact.

decrease of the film specific wear rate leads always to a decrease of the wear depth. The obtained results reveal that a reduction on wear depth of almost four times could be obtained by the deposition of a $4 \mu \mathrm{m}$ coating with a specific wear rate 20 times lower than the substrate.

\section{Demonstration of the model}

To validate the model a micro-abrasion study was carried out using coated cylindrical specimens, with nominal diameters of $8 \mathrm{~mm}$. The specifications and procedures of the experimental equipment were explained elsewhere [1]. The rotating specimen used was a DIN Ck45 steel shaft with a diameter of $15 \mathrm{~mm}$ and a hardness of $185 \mathrm{HV} 1$. The abrasive medium is a slurry of SiC particles with P2500 grit, mean size of $8.4 \mu \mathrm{m}$, in distilled water, with a concentration of $10 \mathrm{vol} . \%$ The hardness usually of $\mathrm{SiC}$ is between 24.5 and $28.2 \mathrm{GPa}$ [9].

The coated material tested was a pre-treated $36 \mathrm{CrNiMo} 4$ electroplated with a $15 \mu \mathrm{m}$ film of hard chromium. The hardness of the tested material was measured both on the core and on the surface obtaining, respectively, $285 \mathrm{HV} 1$ and $1250 \mathrm{HV} 0.05$. The normal load and the rotational speed were kept constant during the study, with the respective, values of $2 \mathrm{~N}$ and $100 \mathrm{rpm}$ (sliding speed of $0.078 \mathrm{~m} / \mathrm{s}$ ). To characterize the coated specimens a set of tests were carried out keeping constant all the test parameter except the duration. The numbers of rotations con- 


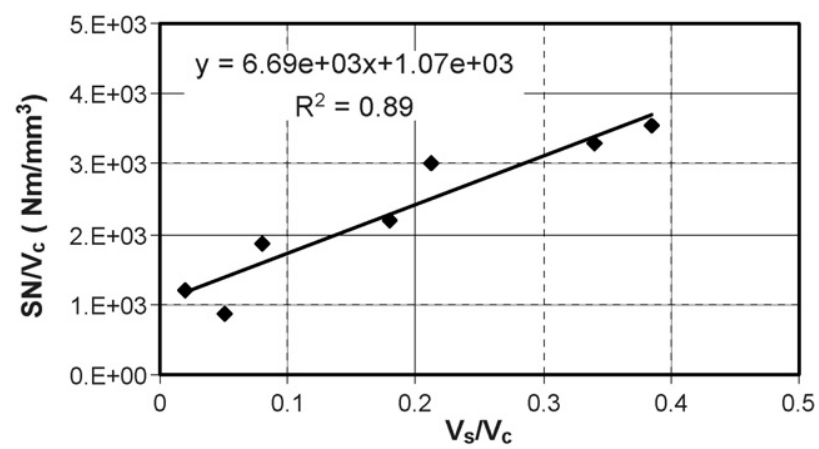

Fig. 5. Application of the Allsop method to the separation of $k_{\mathrm{S}}$ and $k_{\mathrm{c}}$.

sidered were: $8,15,20,30,40,100,200,300,400$ and 500 . Three tests were conducted for each condition, and the average value was used in the results. Before and after the test, the samples were been ultrasonically cleaned with acetone to remove all traces of contaminants. A Philips XL30-TMP scanning electron microscope was used to measure the wear scars and to observe the morphology of the wear surfaces.

For the tests above 20 rotations the substrate was reached and Eq. (5) was applied to calculate volume removed from the substrate and also the total volume of removed material. Therefore, as described elsewhere [1], the values of wear volume could be achieved for the substrate and for the coating, respectively, $V_{\mathrm{s}}$ and $V_{\mathrm{c}}$.

Allsop [10], derived a method that allows the separation of the specific wear rates for both substrate and coating from only one set of ball-cratering tests of flat thin-coated surface. At least, the basic principles are the same for both ball-cratering and for cross-cylinder micro-abrasion; therefore, the Allsop procedure will be applied to analyse the results. The Allsop method rearrange the Eq. (2) in order to separate the values of the specific wear rates of the substrate and of the coating, respectively, $k_{\mathrm{s}}$ and $k_{\mathrm{c}}$, obtaining the following equation:

$\frac{S N}{V_{\mathrm{c}}}=\left(\frac{V_{\mathrm{s}}}{V_{\mathrm{c}}}\right) \frac{1}{k_{\mathrm{s}}}+\frac{1}{k_{\mathrm{c}}}$

Fig. 5 shows the application of Allsop method to analysing the results and a suitable correlation was obtained. The $k_{\mathrm{s}}$ and $k_{\mathrm{c}}$ values are calculated, respectively as the inverse values of the slope and the intercept of the linearization of $S N / V_{\mathrm{c}}$ as function of $V_{\mathrm{s}} / V_{\mathrm{c}}$. The results obtained for the tested material were $1.49 \times 10^{-4}$ and $9.34 \times 10^{-4} \mathrm{~mm}^{3} / \mathrm{Nm}$, respectively, for $k_{\mathrm{S}}$ and $k_{\mathrm{c}}$.

To demonstrate the reliability of the prediction method, the obtained specific wear rates have been used to predict the evolution of the wear volumes and also the wear depth. Fig. 6 compares the prediction to the experimental results, the lines represent the forecast values and the points correspond to experimental results. A very good agreement between experimental and predicted values has been achieved both for wear volumes and wear depth. The good correlation between experimental and predicted values justifies the ability of the developed models.
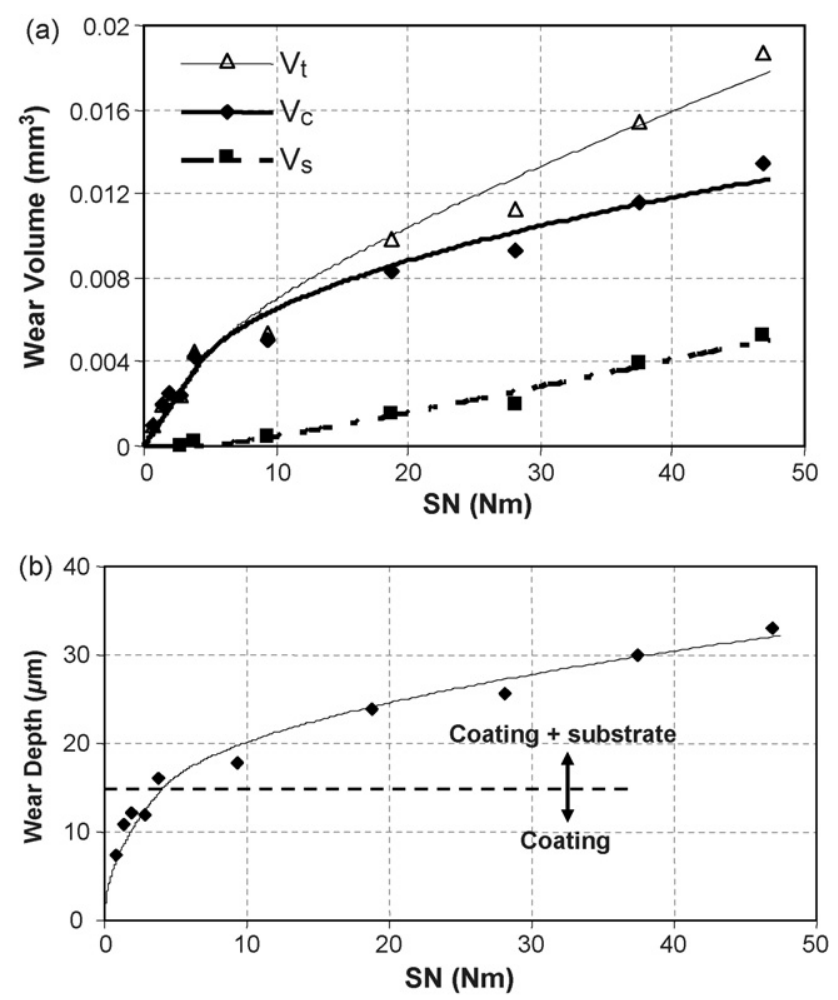

Fig. 6. Comparison of the experimental results with the values predicted by the model developed.

\section{Concluding remarks}

Although coatings are subject to single wear processes in few applications, the abrasion resistance is indubitably relevant to be used together other material properties in material selection. Therefore, the possibility to transpose directly the results of the proposed model to practical applications is very small. However, the main advantage of the method is the possibility to compare different kind of coatings in order to forecast their duration if it is assumed that the abrasion is the unique failure mode. It is expected that the model could be useful in the preliminary tasks of engineering design, to compare different surface engineering solutions from the abrasion point of view.

To demonstrate the suitability of the geometrical model for characterising the wear behaviour of coated cylindrical surfaces, the forecast wear evolutions were compared to the results of experiments and very good correlations were reached.

\section{References}

[1] A. Ramalho, Micro-abrasive wear test of thin coated cylindrical surfaces, Tribol. Lett. 16 (1-2) (2004) 133-141.

[2] A. Ramalho, Micro-scale abrasive wear of coated surfaces-prediction models, Surf. Coat. Technol. 197 (2005) 358-366.

[3] K. Holmberg, A. Laukkanen, H. Ronkainen, K. Wallin, S. Varjus, J. Koskinen, Tribological contact analysis of a rigid ball sliding on a hard coated surface. Part I. Modelling stresses and strains, Surf. Coat. Technol. 200 (12-13) (2006) 3793-3809.

[4] K. Holmberg, A. Laukkanen, H. Ronkainen, K. Wallin, S. Varjus, J. Koskinen, Tribological contact analysis of a rigid ball sliding on a hard coated surface. Part II. Material deformations, influence of coating thickness and Young's modulus, Surf. Coat. Technol. 200 (12-13) (2006) 3810-3823. 
[5] A. Kassman, S. Jacobson, P. Hedenqvist, M. Olsson, A new test method for the intrinsic abrasion resistance of thin coatings, Surf. Coat. Technol. 50 (1991) 75-84.

[6] K.L. Rutherford, I.M. Hutchings, A micro-abrasive wear test, with particular application to coated systems, Surf. Coat. Technol. 79 (1996) 231-239.

[7] H. Czichos, Tribology: A Systems Approach to the Science and Technology of Friction, Lubrication, and Wear, Elsevier Scientific Pub Co.
[8] Y. Kusano, K. Van Acker, I.M. Hutchings, Methods of data analysis for the micro-scale abrasion test on coated substrates, Surf. Coat. Technol. 183 (2004) 312-327.

[9] H.O. Pierson, Handbook of Refractory Carbides and Nitrides, Noyes Publications, New Jersey, USA, 1996.

[10] D.N. Allsop, Abrasive wear of bulk materials and hard coatings, PhD thesis, University of Cambridge, 1999. 